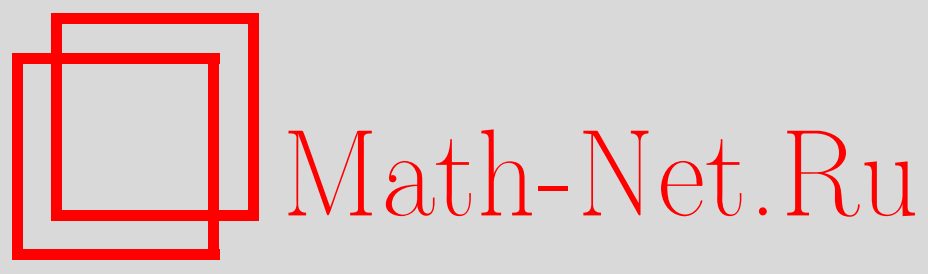

С. П. Чулков, О сходимости формальных решений систем дифференциальных уравнений в частных производных, Функи. анализ и его прил., 2005, том 39, выпуск 3, 64-75

DOI: https://doi.org/10.4213/faa75

Использование Общероссийского математического портала Math-Net.Ru подразумевает, что вы прочитали и согласны с пользовательским соглашением

http://www . mathnet.ru/rus/agreement

Параметры загрузки:

IP: 3.95 .254 .165

26 апреля 2023 г., 14:05:57

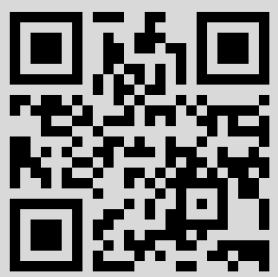




\title{
О сходимости формальных решений систем дифференциальных уравнений в частных производных*
}

\author{
(c) 2005. С. П. Чулков
}

\section{§1. Введение}

В работе исследуется один из вариантов классического вопроса о сходимости формальных решений систем дифференциальных уравнений в частных производных. В теории обыкновенных дифференциальных уравнений рассматривают уравнения, разрешенные относительно производных старшего порядка. Для аналитических обыкновенных дифференциальных уравнений задача Коши всегда разрешима, и всякое формальное решение является аналитическим. Одним из многомерных обобщений этого утверждения является теорема КошиКовалевской. Эта теорема, в частности, описывает все формальные решения уравнений типа Коши-Ковалевской и выясняет, когда эти решения сходятся. Для уравнений этого типа, как и для обыкновенных дифференциальных уравнений, выделена частная производная, которая является старшей.

Рикье в работе [1] построил общую теорию, содержащую теорему КошиКовалевской как частный случай. В многомерном случае нет априори выделенной производной, которую можно было бы рассматривать как старшую. Рикье задается вопросом, какие частные производные неизвестной функции следует назвать главными и как правильно задавать начальные данные, чтобы была выполнена теорема существования и единственности для аналитических и формальных решений. В своей замечательной работе Рикье впервые вводит полное упорядочение на множестве частных производных функции многих переменных и, используя его, получает серьезное продвижение в поставленном вопросе. В случае линейных систем с постоянными коэффициентами метод Рикье, по существу, содержит в себе то, что впоследствии стало называться базисами Грёбнера и произвело революцию в вычислительных аспектах коммутативной алгебры. Основное внимание в работе Рикье уделено вопросу правильного задания начальных данных и вопросу построения соответствующих им формальных решений. Рикье выясняет вопрос о сходимости этих формальных решений. Все же в работе Рикье в силу большой общности поставленных задач ответы не доведены до конца: метод Рикье применим к системам уравнений, разрешенным относительно старших производных, только при выполнении некоторых весьма ограничительных и сложно проверяемых условий на исследуемую систему уравнений.

Изложение работы Рикье на русском языке содержится в книге С. П. Финикова [2].

* Во время выполнения данной работы автор получал частичную финансовую поддержку по грантам РФФИ-01-01-00739 и НШ-1972.2003.1. 
Наиболее полные теоремы существования и единственности для формальных и аналитических решений удается получить в случае систем линейных дифференциальных уравнений с аналитическими коэффициентами. Исследование случая линейных систем было проведено В. П. Паламодовым в работе [3]. Паламодов следует идее Рикье. Ему удалось с помощью алгебраической техники найти новые формальные решения, которые не были известны Рикье (см. [4]). Доказанная в [3] теорема существования и единственности содержит утверждение о сходимости формальных решений, которое не покрывается теоремой сходимости Рикье. Доказательство сходимости формальных решений у Паламодова опирается на разработанную им технику работы с линейными дифференциальными операторами и не обобщается на случай нелинейных систем.

Вопрос построения формальных решений по сути алгебраический (см. [4,5]), и мы здесь им не занимаемся. Цель данной работы состоит в исследовании сходимости заданных формальных решений (найденных любым способом). Наша теорема сходимости применима к любым системам дифференциальных уравнений в частных производных, разрешенным относительно старших производных, и, что особенно важно, к любым системам, «почти разрешенным относительно старших производных» (см. ниже). В ней утверждается, что формальный ряд, являющийся формальным решением системы, сходится, если и только если сходится определенная частичная сумма этого ряда. Наша теорема обобщает теорему сходимости Рикье. Доказательство опирается на некоторые идеи его работы [1]. Но наше обобщение применимо к формальным решениям «паламодовского типа» (которые бывают у систем, «почти разрешенных относительно старших производных»), о которых Рикье не знал и к которым его метод не применим. Наша теорема обобщает также следствия работы Паламодова [3], касающиеся сходимости формальных решений: в отличии от результатов Паламодова она применима к нелинейным системам.

Основная теорема данной работы допускает естественное обобщение на случай систем с несколькими неизвестными функциями (см. §5).

Кроме работ Паламодова и Рикье, отметим работы Овсянникова [6], Пэйта [7], Тревиса [8], Ниренберга [9] и Нишиды [10], содержащие различные варианты доказательства и обобщения теоремы Коши-Ковалевской.

Материал распределен по параграфам следующим образом. В $§ 2$ даются необходимые определения и формулируется основная теорема. В §3 доказываются необходимые свойства упорядоченной полугруппы $\mathbb{Z}_{\geqslant 0}^{n}$. 44 посвящен доказательству основной теоремы. В $\S 5$ разбираются примеры и приводятся заключительные замечания.

Автор очень благодарен А. Г. Хованскому за постановку задачи, многочисленные обсуждения и постоянное внимание к работе и М. А. Шубину за ценные замечания.

\section{§2. Формулировка результата}

Рассмотрим полугруппу $\mathbb{Z}_{\geqslant 0}^{n}=\left\{\left(\alpha_{1}, \ldots, \alpha_{n}\right) \mid \alpha_{i} \in \mathbb{Z}, \alpha_{i} \geqslant 0\right\}$. Модулем элемента $\alpha$ полугруппы назовем неотрицательное целое число $|\alpha|$, равное сумме $\sum \alpha_{i}$.

Фиксируем на полугруппе $\mathbb{Z}_{\geqslant 0}^{n}$ отношение порядка $\prec$, удовлетворяющие следующим условиям: 
(а) для любых элементов $\alpha$ и $\beta$ полугруппы, модули которых удовлетворяют неравенству $|\alpha|<|\beta|$, верно неравенство $\alpha \prec \beta$;

(b) отношение $\prec$ согласовано с операцией сложения на $\mathbb{Z}_{\geqslant 0}^{n}$, т. е. для любых элементов полугруппы $\alpha, \beta$ и $\gamma$ неравенство $\alpha \prec \beta$ влечет за собой неравенство $\alpha+\gamma \prec \beta+\gamma$.

Условие (а) гарантирует, что $\left(\mathbb{Z}_{\geqslant 0}^{n}, \preceq\right)$ есть вполне упорядоченное множество.

В качестве $\prec$, например, можно рассмотреть следующее упорядочение. Для произвольных элементов $\alpha$ и $\beta$ полугруппы $\mathbb{Z}_{\geqslant 0}^{n}$ мы сравниваем их модули, а если модули равны, сравниваем эти элементы лексикографически.

В окрестности точки 0 пространства $\mathbb{C}^{n}$ с координатами $x_{1}, \ldots, x_{n}$ рассмотрим следующую конечную систему дифференциальных соотношений:

$$
\left\{\begin{array}{c}
\partial_{\gamma_{1}} z=F_{1}\left(x, \partial_{\alpha} z\right)+M_{1}\left(x, \partial_{\alpha} z\right), \\
\ldots \ldots \ldots \ldots \ldots \ldots \ldots \ldots \ldots \ldots \ldots \ldots \ldots \ldots \ldots \ldots \\
\partial_{\gamma_{k}} z=F_{k}\left(x, \partial_{\alpha} z\right)+M_{k}\left(x, \partial_{\alpha} z\right),
\end{array}\right.
$$

где $F_{1}, \ldots, F_{k}$ и $M_{1}, \ldots, M_{k}$ - голоморфные функции переменных $x_{1}, \ldots, x_{n}$ и производных $\partial_{\alpha} z$ функции $z$. Здесь и далее через $\partial_{\alpha}$, где $\alpha \in \mathbb{Z}_{\geqslant 0}^{n}$, обозначен оператор дифференцирования $\partial^{|\alpha|} / \partial x_{1}^{\alpha_{1}} \cdots \partial x_{n}^{\alpha_{n}}$.

Пусть формальный ряд $z=\sum_{\alpha \in \mathbb{Z}_{\geqslant 0}^{n}} z_{\alpha} x^{\alpha}$, где $z_{\alpha}-$ комплексные числа и через $x^{\alpha}$ обозначен моном $x_{1}^{\alpha_{1}} \cdots x_{n}^{\alpha_{n}}$, является формальным решением данной системы.

Предположим, кроме того, что выполнены следующие условия:

1) для каждого $i$ функция $F_{i}$ зависит от переменных $x_{1}, \ldots, x_{n}$ и только от тех производных $\partial_{\alpha} z$, для показателей $\alpha$ которых выполнено неравенство $\alpha \prec \gamma_{i}$;

2) для каждого $i$ функция $M_{i}$ имеет вид

$$
M_{i}\left(x, \partial_{\alpha} z\right)=\sum_{\beta,|\beta|=\left|\gamma_{i}\right|} M_{i}^{\beta}\left(x, \partial_{\alpha} z\right) \partial_{\beta} z,
$$

где $M_{i}^{\beta}$ - голоморфные функции переменных $x_{1}, \ldots, x_{n}$ и производных $\partial_{\alpha} z$, таких, что $|\alpha|<\left|\gamma_{i}\right|$, причем в начальной точке для любых допустимых $i$ и $\beta$

$$
M_{i}^{\beta}\left(0, \alpha ! z_{\alpha}\right)=0
$$

здесь и далее через $\alpha$ ! обозначено произведение факториалов $\alpha_{1} ! \cdots \alpha_{n}$ !.

Говоря неформально, данные условия означают что наша система записана в виде, «почти разрешенном относительно старших, в смысле нашего упорядочения, производных», с той лишь разницей, что в правую часть каждого уравнения разрешено добавить линейную относительно производных старшего (наибольшего) порядка функцию (для каждого уравнения свою), коэффициенты которой обращаются в нуль в начальной точке. В каждом уравнении некоторые из добавленных в правую часть производных могут быть больше в смысле нашего упорядочения, чем производная, стоящая в левой части уравнения.

Назовем октантом $O^{n}(a)$ с вершиной в точке $a$ полугруппы $\mathbb{Z}_{\geqslant 0}^{n}$ множество $\left\{\alpha \in \mathbb{Z}_{\geqslant 0}^{n} \mid\right.$ существует $\beta \in \mathbb{Z}_{\geqslant 0}^{n}$, такой, что $\left.\alpha=a+\beta\right\}$.

Рассмотрим подмножество $I=\bigcup_{i=1}^{k} O\left(\gamma_{i}\right)$ полугруппы $\mathbb{Z}_{\geqslant 0}^{n}$. В сделанных выше предположениях верна следующая 
ТЕОремА. Предположим, что укорочение $\tilde{z}(x)=\sum_{\alpha \in \mathbb{Z}_{\geqslant 0}^{n} \backslash I} z_{\alpha} x^{\alpha}$ ряда z имеет ненулевой радиус сходимости. Тогда и формальное решение z имеет ненулевой радиус сходимости.

Доказательство теоремы приведено в 44 .

ЗАмЕчАНИЕ. Отметим, что условие (1) линейности функций $M_{i}$ относительно производных старшего порядка не слишком сужает круг рассматриваемых систем. Действительно, если изначально некоторая функция $M_{i}$ не была линейной относительно производных старшего порядка, то, продифференцировав соответствующее уравнение вдоль любой переменной, мы получим новое уравнение, в котором новая функция $M_{i}$ уже будет линейной относительно производных старшего порядка, а другие условия не нарушатся.

\section{§3. Упорядоченная полугруппа $\mathbb{Z}_{\geqslant 0}^{n}$}

Напомним, что в предыдущем параграфе мы фиксировали некоторое полное упорядочение на полугруппе $\mathbb{Z}_{\geqslant 0}^{n}$. Оказывается, что, каким бы ни был порядок $\prec$ на полугруппе $\mathbb{Z}_{\geqslant 0}^{n}$, на любом конечном подмножестве этой полугруппы он задается одним линейным функционалом. А именно, для доказательства основной теоремы потребуется следующая

Лемма 1. Пусть $A-$ конечное подмножсество полугруппы $\mathbb{Z}_{\geqslant 0}^{n}$. Существует линейный функиионал

$$
\Pi_{A}: \mathbb{Z}_{\geqslant 0}^{n} \rightarrow \mathbb{R}_{\geqslant 0}, \quad \Pi_{A}(\alpha)=\sum_{i=1}^{n} \pi_{i} \alpha_{i},
$$

где $\pi_{i}$ - некоторые положсттельные вещественные числа $и \alpha=\left(\alpha_{1}, \ldots, \alpha_{n}\right) \in$ $\mathbb{Z}_{\geqslant 0}^{n}$, удовлетворяющий следующему условию:

для любых $\alpha, \beta \in A$, таких, что $\alpha \prec \beta$, выполнено неравенство

$$
\Pi_{A}(\alpha)<\Pi_{A}(\beta) \text {. }
$$

ДокАЗАтельство. Рассмотрим цепочку естественных вложений $\mathbb{Z}_{\geqslant 0}^{n} \subset \mathbb{Z}^{n}$ $\subset \mathbb{R}^{n}$. Обозначим через $B$ следующее конечное подмножество группы $\mathbb{Z}^{n}$ :

$$
B=\left\{\delta \in \mathbb{Z}^{n} \mid \exists \alpha, \beta \in A: \alpha \prec \beta, \delta=\beta-\alpha\right\} .
$$

Пусть $\operatorname{conv}(B)$ - выпуклая оболочка точек множества $B$ в пространстве $\mathbb{R}^{n}$. Так как упорядочение $\prec$ согласовано с операцией сложения на полугруппе $\mathbb{Z}_{\geqslant 0}^{n}$, то $\operatorname{conv}(B)$ не содержит точки 0. Действительно, предположим противное. Пусть

$$
\sum_{1}^{N} p_{i} \delta^{i}=0
$$

где $\delta^{i} \in B \subset \mathbb{Z}^{n}\left(\delta^{i}=\beta^{i}-\alpha^{i}, \beta^{i}, \alpha^{i} \in A\right)$ и $p_{i} \in \mathbb{R}, p_{i}>0$. Но (3) можно рассматривать как систему линейных однородных уравнений с целыми коэффициентами на координаты вектора $p=\left(p_{1}, \ldots, p_{N}\right) \in \mathbb{R}^{N}$. Существование нетривиального решения системы влечет за собой существования нетривиального векторного подпространства решений. Так как коэффициенты уравнений систе- 
мы (3) - целые числа, то рациональные векторы всюду плотны в пространстве решений. Следовательно, существуют рациональные - а значит, и целые положительные числа $\tilde{p}_{i}$, такие, что

$$
\sum_{1}^{N} \tilde{p}_{i} \delta^{i}=0 .
$$

Тогда

$$
\sum_{1}^{N} \tilde{p}_{i} \beta^{i}=\sum_{1}^{N} \tilde{p}_{i} \alpha^{i}
$$

$\mathrm{C}$ другой стороны, $\alpha_{i} \prec \beta_{i}$, а следовательно, $\sum_{1}^{N} \tilde{p}_{i} \alpha_{i} \prec \sum_{1}^{N} \tilde{p}_{i} \beta_{i}$. Противоречие. Так как замкнутое ограниченное выпуклое множество $\operatorname{conv}(B)$ не содержит точки 0, существует функционал

$$
L: \mathbb{R}^{n} \rightarrow \mathbb{R}, \quad L(x)=\sum_{i=1}^{n} l_{i} x_{i},
$$

такой, что $L(x)>0$ для любой точки $x \in \operatorname{conv}(B)$. Положив теперь $\pi_{i}=S+l_{i}$, где $S$ - достаточно большое натуральное число, получим искомый функционал $\Pi_{A}$.

ЗАмечАниЕ. Рассуждения, используемые выше, нетрудно продолжить до доказательства следующего хорошо известного факта (см., например, [11] или $[12])$.

ПРЕДЛОЖЕНИЕ. Рассмотрим на полугруппе $\mathbb{Z}_{\geqslant 0}^{n}$ отношение порядка $\prec$, согласованное с операцией сложения. Тогда существуют линейные функииональ $\Pi^{1}, \ldots, \Pi^{j}$, где $j \leqslant n$,

$$
\Pi^{i}: \mathbb{Z}_{\geqslant 0}^{n} \mapsto \mathbb{R},
$$

такие, что порядок является лексикографическим относительно этого набора функиионалов. То есть утвержсдение $\alpha \prec \beta$ равносильно утвержсдению

$$
\Pi^{1}(\alpha)=\Pi^{1}(\beta), \ldots, \quad \Pi^{i}(\alpha)=\Pi^{i}(\beta), \quad \Pi^{i+1}(\alpha)<\Pi^{i+1}(\beta)
$$

для некоторого $i$, принадлежащего множеству $\{0, \ldots, j-1\}$.

Обозначим через $\Pi_{k}$, где $k$ - натуральное число, функционал, удовлетворяющий условиям леммы 1 для множества $A_{k}=\left\{\alpha \in \mathbb{Z}_{\geqslant 0}^{n}:|\alpha| \leqslant k\right\}$. Пусть $\mu_{k}=\min _{\left\{\alpha, \beta \in A_{k}\right\}}\left|\Pi_{k}(\beta)-\Pi_{k}(\alpha)\right|$. Отметим, что $\mu_{k}>0$.

\section{§4. Доказательство теоремы}

Наше доказательство основано на методе мажорант. Рассмотрим кольцо $\mathbb{C}\left[\left[y_{1}, \ldots, y_{l}\right]\right]$ формальных рядов от некоторых переменных $y_{1}, \ldots, y_{l}$. Пусть $A, B \in \mathbb{C}\left[\left[y_{1}, \ldots, y_{l}\right]\right]$.

ОПрЕДЕЛЕНИЕ. Будем говорить, что формальный ряд $A(y)=\sum_{\alpha \in \mathbb{Z}_{\geqslant 0}^{l}} a_{\alpha} y^{\alpha}$ усиливает (мажсорирует) ряд $B(y)=\sum_{\alpha \in \mathbb{Z}_{\geqslant 0}^{l}} b_{\alpha} y^{\alpha}$, если для любого элемента $\alpha$ полугруппы $\mathbb{Z}_{\geqslant 0}^{l}$ выполнены следующие два условия:

$$
a_{\alpha} \in \mathbb{R}_{\geqslant 0} \quad \text { и } \quad\left|b_{\alpha}\right| \leqslant a_{\alpha} .
$$


Идея доказательства заключается в построении сходящегося ряда, мажорирующего данное формальное решение. Точнее, этот мажорирующий ряд строится по решению некоторого уравнения, грубо говоря, мажорирующего каждое уравнение исходной системы. Мажорирующее уравнение оказывается обыкновенным дифференциальным уравнением, для доказательства существования решения которого применима стандартная теорема существования и единственности.

В разд. 4.1 доказываются необходимые подготовительные леммы о мажорировании.

В разд. 4.2-4.5 теорема доказывается для частного случая - уравнения системы линейны относительно производных старшего порядка, а главные производные имеют один и тот же порядок. В разд. 4.2 формулируются условия разбираемого частного случая. В разд. 4.3 система с помощью замены координат приводится к виду, по которому легко выписать нужное нам мажорирующее уравнение. В разд. 4.4 мы предъявляем мажорирующее уравнение и устанавливаем существование аналитического решения этого уравнения. В разд. 4.5 мы строим по решению мажорирующего уравнения некоторый сходящийся ряд и, опираясь на лемму 2 разд. 4.1, доказываем, что полученный ряд мажорирует исходное формальное решение системы. И, наконец, в разд. 4.6 мы завершаем доказательство теоремы, сводя общий случай к разобранному.

4.1. Леммы о мажорировании. В первой из двух лемм данного раздела формулируется необходимый нам вариант утверждения о том, что свойство мажорирования выдерживает операцию композиции.

Рассмотрим голоморфные функции $f_{1}$ и $f_{2}$, определенные в окрестности нуля пространства $\mathbb{C}^{n+m}=\left\{\left(x_{1}, \ldots, x_{n}, \xi_{1}, \ldots, \xi_{m}\right) \mid x_{i}, \xi_{j} \in \mathbb{C}\right\}$. Предположим, что разложение в ряд функции $f_{2}$ усиливает разложение функции $f_{1}$.

Фиксируем набор $\alpha_{1} \prec \cdots \prec \alpha_{m} \prec \alpha_{0}$ из $m+1$ элементов полугруппы $\mathbb{Z}_{\geqslant 0}^{n}$.

Пусть, далее, $w=\sum w_{\alpha} x^{\alpha} \in \mathbb{R}_{\geqslant 0}[[x]]$ и $z=\sum z_{\alpha} x^{\alpha} \in \mathbb{C}[[x]]-$ некоторые ряды, и предположим, что выполнены следующие два условия:

$$
\begin{aligned}
& \left|z_{\alpha}\right| \leqslant w_{\alpha} \text { для всех } \alpha \prec \alpha_{0}, \\
& w_{\alpha_{i}}=z_{\alpha_{i}}=0 \text { для любого } i(1 \leqslant i \leqslant m) .
\end{aligned}
$$

В этом случае корректно определены ряды $W \in \mathbb{R}_{\geqslant 0}[[x]]$, результат подстановки в разложение функции $f_{2}$ вместо переменных $\xi_{i}$ рядов $\partial_{\alpha_{i}} w(1 \leqslant i \leqslant m)$, и $Z \in \mathbb{C}[[x]]$, результат подстановки в разложение функции $f_{1}$ вместо переменных $\xi_{i}$ рядов $\partial_{\alpha_{i}} z(1 \leqslant i \leqslant m)$.

ЛЕмма 2. Для любого $\beta \prec \alpha_{0}$ выполняется неравенство $\left|\partial_{\beta} Z\right|_{0}\left|\leqslant \partial_{\beta} W\right|_{0}$.

ДокАзАтельство. Пусть $Z=\sum_{\alpha} Z_{\alpha} x^{\alpha}$ и $W=\sum_{\alpha} W_{\alpha} x^{\alpha}$. Тогда $\left.\partial_{\beta} Z\right|_{0}=$ $\beta ! Z_{\beta}$ и $\left.\partial_{\beta} W\right|_{0}=\beta ! W_{\beta}$ суть суммы по одному и тому же набору индексов произведений вида

$$
\beta ! f_{(\alpha, \delta)}^{1} \prod_{i=1}^{m}\left(\alpha_{i} !\right)^{\delta_{i}} z_{\theta_{1}+\alpha_{i}} \cdots z_{\theta_{\delta_{i}}+\alpha_{i}}
$$

$$
\beta ! f_{(\alpha, \delta)}^{2} \prod_{i=1}^{m}\left(\alpha_{i} !\right)^{\delta_{i}} w_{\theta_{1}+\alpha_{i}} \cdots z_{\theta_{\delta_{i}}}+\alpha_{i}
$$


соответственно; в этих формулах $\alpha, \theta_{j} \in \mathbb{Z}_{\geqslant 0}^{n}, \delta=\left(\delta_{1}, \ldots, \delta_{m}\right) \in \mathbb{Z}_{\geqslant 0}^{m}$, а через $f_{(\alpha, \delta)}^{j}, j=1,2$, обозначены коэффициенты разложений в ряды функций $f^{j}$. Отметим, что $\alpha+\sum \theta_{i}=\beta \prec \alpha_{0}$ в формулах (4) и (5), а следовательно, в силу условий, сформулированных выше, каждое слагаемое в (4) больше или равно модулю соответствующего слагаемого в (5). Отсюда получаем утверждение леммы 2.

Доказательство следующей несложной леммы можно найти в [2].

ЛЕмма 3. Предположим, что ряд $A(x)=\sum_{\alpha \in \mathbb{Z}_{\geqslant 0}^{n}} a_{\alpha} x^{\alpha}$ абсолютно сходится в точке $x_{1}=\cdots=x_{n}=\rho>0$ и $M$ - положительное число, большее абсолютной величинь любого члена ряда $A(\rho)$. Тогда разложсения в степенной ряд в окрестности нуля функиий

$$
F_{1}(x)=\frac{M}{\left(1-x_{1} / \rho\right) \ldots\left(1-x_{n} / \rho\right)} \quad u \quad F_{2}(x)=\frac{M}{\left(1-\left(x_{1}+\cdots+x_{n}\right) / \rho\right)}
$$

усиливают ряд $A(x)$.

4.2. Формулировка условий частного случая. Без ограничения общности можно считать коэффициенты $z_{\alpha}$ ряда $z$ при $\alpha \in \mathbb{Z}_{\geqslant 0}^{n} \backslash I$ равными 0. Действительно, по условию ряд, естественным образом из них составленный, определяет некоторую аналитическую функцию $\varphi$ в окрестности начала координат. Рассматривая новую неизвестную функцию $\tilde{z}=z-\varphi$, получаем требуемое.

Разберем теперь следующий частный случай. Предположим, что наша система линейна относительно производных старшего порядка, а все главные производные (т. е. производные, стоящие в левых частях уравнений) имеют один и тот же порядок. Точнее, соотношения имеют следующий вид:

$$
\left\{\begin{array}{c}
\partial_{\gamma_{1}} z=\sum_{|\alpha|=N, \alpha \prec \gamma_{1}} f_{\alpha}^{1} \partial_{\alpha} z+f^{1}+\sum_{|\alpha|=N} M_{1}^{\alpha} \partial_{\alpha} z, \\
\ldots \ldots \ldots \ldots \ldots \ldots \ldots \ldots \ldots \ldots \ldots \ldots \ldots \ldots \ldots \ldots \ldots \ldots \ldots \\
\partial_{\gamma_{k}} z=\sum_{|\alpha|=N, \alpha \prec \gamma_{k}} f_{\alpha}^{k} \partial_{\alpha} z+f^{k}+\sum_{|\alpha|=N} M_{k}^{\alpha} \partial_{\alpha} z,
\end{array}\right.
$$

где $\left|\gamma_{1}\right|=\cdots=\left|\gamma_{k}\right|=N>0$, а голоморфные функции $f_{\alpha}^{i}, f^{i}, M_{k}^{\alpha}$ зависят от переменных $x_{1}, \ldots, x_{n}$ и производных $\partial_{\beta} z$, таких, что $|\beta|<N$. Кроме того, по-прежнему $M_{i}^{\alpha}(0)=0$ для всех допустимых $i$ и $\alpha$.

4.3. Замена координат. В этом разделе с помощью замены координат мы добиваемся того, чтобы старшие производные стали главными. При данной замене коэффициенты при остальных производных умножаются на некоторые малые числа.

В сделанных предположениях $f_{\alpha}^{i}, f^{i}, M_{i}^{\alpha}$ - голоморфные функции своих переменных в окрестности нуля. Для всех допустимых значений параметров $i$ и $\alpha$ разложим функции $f_{\alpha}^{i}, f^{i}$ и $M_{i}^{\alpha}$ в степенные ряды в окрестности нуля. Предположим, что все эти ряды абсолютно сходятся в точке $x_{1}=\cdots=x_{n}=\partial_{\alpha} z=\rho$, где параметр $\alpha$ пробегает подмножество полугруппы $\mathbb{Z}_{\geqslant 0}^{n}$, выделяемое условием $|\alpha|<N$. В силу леммы 3 разд. 4.1 выберем положительную вещественную 
константу $C$ так, чтобы разложение функции

$$
\frac{C}{\left(1-\left(x_{1}+\cdots+x_{n}+\sum_{|\alpha|<N} \partial_{\alpha} z\right) / \rho\right)}
$$

усиливало соответствующие разложения функций $f_{\alpha}^{i}, f^{i}, M_{i}^{\alpha}$ для всех допустимых значений $i$ и $\alpha$.

Обозначим через П функционал $\Pi_{N}$ (см. §3), и пусть $\mu=\mu_{N}$. Рассмотрим положительное вещественное число $\theta<1$, такое, что

$$
\theta^{\mu}<\varepsilon=\frac{1}{2} \frac{1}{\Delta_{N} C},
$$

где через $\Delta_{i}\left(i \in \mathbb{Z}_{\geqslant 0}\right)$ мы обозначаем количество элементов $\alpha$ полугруппы $\mathbb{Z}_{\geqslant 0}^{n}$, таких, что $|\alpha|=i$.

Положим $y_{i}=\theta^{-\pi_{i}} x_{i}, 1 \leqslant i \leqslant n$. Тогда

$$
\frac{\partial^{|\alpha|} z}{\partial x^{\alpha}}(x)=\theta^{-\Pi(\alpha)} \frac{\partial^{|\alpha|} z}{\partial y^{\alpha}}(y(x)) .
$$

После замены координат и деления на коэффициент при старшей производной уравнения (6) примут вид

$$
\begin{aligned}
\partial_{\gamma_{i}} z= & \sum_{|\alpha|=N} f_{\alpha}^{i}\left(y, \theta^{-\Pi(\gamma)} \partial_{\gamma} z(y)\right) \theta^{\Pi\left(\gamma_{i}\right)-\Pi(\alpha)} \partial_{\alpha} z+f^{i}\left(y, \theta^{-\Pi(\beta)} \partial_{\beta} z(y)\right) \theta^{\Pi\left(\gamma_{i}\right)} \\
& +\sum_{|\alpha|=N} M_{i}^{\alpha}\left(y, \theta^{-\Pi(\gamma)} \partial_{\gamma} z(y)\right) \theta^{\Pi\left(\gamma_{i}\right)-\Pi(\alpha)} \partial_{\alpha} z
\end{aligned}
$$

для каждого $i$. Здесь и далее оператор взятия частной производной рассматривается в новой системе координат. Формальный ряд $z(y)=z(x(y))$ удовлетворяет дифференциальным соотношениям (7) в новой системе координат (с «тождественно нулевыми начальными условиями»). Для всех допустимых $\alpha, i$ положим

$$
\begin{aligned}
\tilde{f}_{\alpha}^{i}\left(y, \partial_{\gamma} z\right) & =f_{\alpha}^{i}\left(y, \theta^{-\Pi(\gamma)} \partial_{\beta} z\right) \theta^{\Pi\left(\gamma_{i}\right)-\Pi(\alpha)} \\
\tilde{f}^{i}\left(y, \partial_{\gamma} z\right) & =f^{k}\left(y, \theta^{-\Pi(\gamma)} \partial_{\gamma} z\right) \theta^{\Pi\left(\gamma_{i}\right)} \\
\widetilde{M}_{i}^{\alpha}\left(y, \partial_{\gamma} z\right) & =M_{i}^{\alpha}\left(y, \theta^{-\Pi(\gamma)} \partial_{\gamma} z\right) \theta^{\Pi\left(\gamma_{i}\right)-\Pi(\alpha)} .
\end{aligned}
$$

По прежнему $\widetilde{M}_{i}^{\alpha}(0)=0$ для всех допустимых значений $i$ и $\alpha$. Докажем следующую лемму.

ЛЕмма 4. Для всех допустимых $i$ и $\alpha$ разложение в степенной ряд функиии

$$
\frac{\varepsilon C}{1-\left(y_{1}+\cdots+y_{n}+\sum_{|\alpha|<N} \partial_{\alpha} z\right) / \rho_{1}}
$$

для некоторого $\rho_{1}, 0<\rho_{1} \ll \rho$, усиливает соответствующие разложения функиий $\tilde{f}_{\alpha}^{i}$ и $\tilde{f}^{i}$, зависящих от переменных $y_{i}$ и некоторых частных производных по этим переменным.

ДокАЗАТЕЛЬСтво. Выберем $\rho_{1}$ следующим образом:

$$
\rho_{1}=\rho \min _{|\alpha| \leqslant N} \theta^{\pi(\alpha)} .
$$


Проверим утверждение леммы для функций $\tilde{f}_{\alpha}^{i}$. Действительно, в координатах $x_{1}, \ldots, x_{n}$ разложение в ряд в нуле функции

$$
\frac{C}{1-\left(x_{1}+\cdots+x_{n}+\sum_{|\alpha|<N} \partial_{\alpha} z\right) / \rho}
$$

усиливает разложение каждой функции $f_{\alpha}^{i}$. Исходя из формул замены координат, мы утверждаем, что в новых координатах разложение в нуле функции

$$
\frac{C}{1-\left(y_{1}+\cdots+y_{n}+\sum_{|\alpha|<N} \partial_{\alpha} z\right) / \rho_{1}}
$$

будет усиливать разложение каждой функции $f_{\alpha}^{i}\left(y, \theta^{-\Pi(\gamma)} \partial_{\beta} z\right)$. Но

$$
\tilde{f}_{\alpha}^{i}\left(y, \partial_{\gamma} z\right)=f_{\alpha}^{i}\left(y, \theta^{-\Pi(\gamma)} \partial_{\beta} z\right) \theta^{\Pi\left(\gamma_{i}\right)-\Pi(\alpha)},
$$

где $\theta^{\Pi\left(\gamma_{i}\right)-\Pi(\alpha)} \leqslant \theta^{\mu}<\varepsilon$, так как $\alpha \prec \gamma_{i}$ и $|\alpha| \leqslant\left|\gamma_{i}\right|=N$. Отсюда получаем требуемое утверждение.

Для функций $\tilde{f}^{i}$ проверка проводится аналогично, с учетом неравенства $\theta^{\gamma_{i}} \leqslant \theta^{\mu}$, верного для каждого $i$ в силу выбора $\mu$.

Выберем положительную вещественную константу $K$ таким образом, чтобы функция

$$
\frac{K C}{1-\left(y_{1}+\cdots+y_{n}+\sum_{|\alpha|<N} \partial_{\alpha} z\right) / \rho_{1}}
$$

усиливала разложение в ряды в начале координат функций $\widetilde{M}_{i}^{\alpha}$ для всех допустимых $i$ и $\alpha$.

4.4. Построение мажорирующего уравнения. Рассмотрим обыкновенное дифференциальное уравнение

$$
\begin{aligned}
Y^{(N)}(t)= & \frac{\varepsilon C}{1-\left(t+\sum_{j=1}^{N-1} \Delta_{j} Y^{(j)}(t)\right) / \rho_{1}}\left(\Delta_{N} Y^{(N)}(t)+1\right) \\
& +\left(\frac{K C}{1-\left(t+\sum_{j=1}^{N-1} \Delta_{j} Y^{(j)}(t)\right) / \rho_{1}}-K C\right) \Delta_{N} Y^{(N)}(t) .
\end{aligned}
$$

Напомним, что через $\Delta_{j}$ мы обозначаем количество элементов $\alpha$ полугруппы $\mathbb{Z}_{\geqslant 0}^{n}$, таких, что $|\alpha|=j$. Переписав уравнение (10) в виде, разрешенном относительно старшей производной, получим

$$
Y^{(N)}(t)=\frac{2 \varepsilon C}{1-2\left(K C \Delta_{N}+1\right)\left(t+\sum_{j=1}^{N-1} \Delta_{j} Y^{(j)}(t)\right) / \rho_{1}} .
$$

Выше мы учли равенство $\varepsilon \Delta_{N} C=1 / 2$. В силу теоремы существования и единственности решения для обыкновенных дифференциальных уравнений существует и единственно решение $Z(t)$ уравнения (11) (или $(10))$ с начальными условиями $Z^{(0)}=\cdots=Z^{(N-1)}=0$.

Так как разложение в нуле в степенной ряд от переменных $t$ и $Y, Y^{(1)}, \ldots$, $Y^{(N-1)}$ функции

$$
\frac{2 \varepsilon C}{1-2\left(K C \Delta_{N}+1\right)\left(t+\sum_{j=1}^{N-1} \Delta_{j} Y^{(j)}(t)\right) / \rho_{1}}
$$


имеет строго положительные коэффициенты, мы можем утверждать, что разложение решения $Z(t)$ в степенной ряд в окрестности нуля также имеет строго положительные коэффициенты.

Положим

$$
G(Y, t)=\frac{C}{1-\left(t+\sum_{j=1}^{N-1} \Delta_{j} Y^{(j)}(t)\right) / \rho_{1}}
$$

4.5. Построение мажорирующего ряда. Докажем следующий результат.

Лемма 5. Разложение в степенной ряд в окрестности нуля функиии $Z\left(\sum_{i=1}^{n} y_{i}\right)$ усиливает формальное решение $z(y)$. Таким образом, ряд z $(y)$ сходится в некоторой окрестности нуля.

ДоказАтеЛьство. Пусть $Z\left(\sum_{i} y_{i}\right)=\sum_{\alpha \in \mathbb{Z}_{\geqslant 0}^{n}} Z_{\alpha} y^{\alpha}$. Покажем индукцией по $\alpha \in \mathbb{Z}_{\geqslant 0}^{n}$, что выполнено неравенство

$$
\left|z_{\alpha}\right| \leqslant Z_{\alpha}
$$

Действительно, условие (12) выполнено для $\alpha \in \mathbb{Z}_{\geqslant 0}^{n} \backslash I$, а следовательно, и для $\alpha=0$. Пусть теперь (12) верно для всех $\alpha \prec \alpha_{0}$. Докажем неравенство для $\alpha_{0}$. Пусть $\alpha_{0}=\beta+\gamma_{i}$ для некоторого $i(1 \leqslant i \leqslant k)$.

Тогда

$$
\alpha_{0} !\left|z_{\alpha_{0}}\right|=\left.\left|\partial_{\alpha_{0}} z\right|_{y=0}|=|\left(\partial_{\beta}\left(\sum_{|\alpha|=N, \alpha \prec \gamma_{i}} \tilde{f}_{\alpha}^{i} \partial_{\alpha} z+\tilde{f}^{i}+\sum_{|\alpha|=N} \widetilde{M}_{i}^{\alpha} \partial_{\alpha} z\right)\right)\right|_{y=0} \mid .
$$

Далее, в силу леммы 2 и равенства

$$
\partial_{\alpha} F\left(\sum y_{i}\right)=F^{(|\alpha|)}\left(\sum y_{i}\right)
$$

где $F$ - произвольная голоморфная функция, получаем

$$
\begin{aligned}
& \left|\left(\partial_{\beta}\left[\sum_{|\alpha|=N, \alpha \prec \gamma_{i}} \tilde{f}_{\alpha}^{i} \partial_{\alpha} z+\tilde{f}^{i}+\sum_{|\alpha|=N} \widetilde{M}_{i}^{\alpha} \partial_{\alpha} z\right]\right)\right|_{y=0} \mid \\
& \leqslant\left(\partial _ { \beta } \left[\varepsilon G\left(Z, \sum_{i} y_{i}\right)\left(\Delta_{N} Z^{(N)}\left(\sum_{i} y_{i}\right)+1\right)\right.\right. \\
& \left.\left.\quad+\left(K G\left(Z, \sum_{i} y_{i}\right)-K C\right) \Delta_{N} Z^{(N)}\left(\sum_{i} y_{i}\right)\right]\right)\left.\right|_{y=0} .
\end{aligned}
$$

Действительно, каждое слагаемое вида $\tilde{f}_{\alpha}^{i} \partial_{\alpha} z$ мажорируется в силу леммы 4 и предположения индукции слагаемым

$$
\varepsilon G\left(Z, \sum_{i} y_{i}\right) Z^{(N)}\left(\sum_{i} y_{i}\right)
$$

а количество слагаемых вида $\tilde{f}_{\alpha}^{i} \partial_{\alpha} z$ не превосходит числа $\Delta_{N}$. Аналогично, используя лемму 4, выбор константы $K$ (см. (9)) и предположение индукции, 
получаем аналогичные оценки для остальных слагаемых; далее применяем лемму 2. Но в силу (10) и (13)

$$
\begin{aligned}
\left(\partial _ { \beta } \left[\varepsilon G\left(Z, \sum_{i} y_{i}\right)\left(\Delta_{N} Z^{(N)}\left(\sum_{i} y_{i}\right)+1\right)\right.\right. \\
\left.\left.\quad+\left(K G\left(Z, \sum_{i} y_{i}\right)-K C\right) \Delta_{N} Z^{(N)}\left(\sum_{i} y_{i}\right)\right]\right)\left.\right|_{y=0} \\
=\left.\partial_{\alpha_{0}} Z\left(\sum_{i} y_{i}\right)\right|_{y=0}=\alpha_{0} ! Z_{\alpha_{0}} .
\end{aligned}
$$

Отсюда получаем утверждение леммы 5.

4.6. Завершение доказательства теоремы. Для завершения доказательства теоремы осталось заметить, что случай любых дифференциальных соотношений сводится к разобранному выше. Действительно, вместо исходных соотношений можно рассмотреть конечный набор всевозможных их следствий вида

$$
\partial_{\beta} \partial_{\gamma_{i}} z=\partial_{\beta} f_{i}\left(x, \partial_{\alpha} z\right)+\partial_{\beta} M_{i}\left(x, \partial_{\alpha} z\right)
$$

где $|\beta|+\left|\gamma_{i}\right|=N, N$ - достаточно большое $\left(\right.$ например, $\left.N=\max _{i}\left|\gamma_{i}\right|+1\right)$ натуральное число. После этого преобразования мы получим набор дифференциальных соотношений вида (6). Формальный ряд $z(x)$ будет удовлетворять новой системе соотношений. Множество $\mathbb{Z}_{\geqslant 0}^{n} \backslash I$ дополнится конечным набором элементов, что не влияет на сходимость ряда $\tilde{z}(x)$, задающего укорочение ряда $z(x)$.

\section{§5. Примеры и замечания}

5.1. Пример. Случай одного уравнения. Рассмотрим дифференциальное уравнение

$$
\partial_{\gamma} z=F\left(x, \partial_{\alpha} z\right)+M\left(x, \partial_{\alpha} z\right)
$$

Предположим, что оно удовлетворяет всем условиям теоремы. В этом случае очевидно, что существует и единственно формальное решение данного уравнения с начальными данными $\left.\partial_{\alpha} z\right|_{x=0}=\alpha ! z_{\alpha}$, где $\alpha$ пробегает подмножество полугруппы $\mathbb{Z}_{\geqslant 0}^{n} \backslash O(\gamma)$ и $z_{\alpha}$ - произвольные комплексные числа. То есть в качестве начальных данных мы произвольным образом задаем коэффициенты искомого решения при мономах с показателями, принадлежащими подмножеству $\mathbb{Z}_{\geqslant 0}^{n} \backslash O(\gamma)$ полугруппы $\mathbb{Z}^{n}$. Рассматриваемое нами укорочение $\tilde{z}(x)$ формального решения $z(x)$ совпадает с рядом начальных данных. В данном случае теорема гарантирует сходимость формального решения, если ряд начальных данных сходится.

5.2. Пример. Необходимость условий теоремы. Рассмотрим уравнение

$$
(1+i) \frac{\partial^{2}}{\partial x \partial y}=\frac{\partial^{2}}{\partial x^{2}}+i \frac{\partial^{2}}{\partial y^{2}} .
$$

Для данного уравнения условия теоремы не выполняются ни для какого упорядочения, удовлетворяющего условиям (a) и (b) из $\S 2$. В этом случае нетрудно построить нетривиальное формальное решение $z=\sum z_{\alpha} x^{\alpha}$, такое, что выполнено равенство $z_{\alpha}=0$ для $\alpha \in \mathbb{Z}_{\geqslant 0}^{2} \backslash O((1,1))$, но формальный ряд $z=\sum z_{\alpha} x^{\alpha}$ 
имеет нулевой радиус сходимости. Действительно, искомое решение задается следующими условиями:

$z_{(0, n)}=0$ для любого неотрицательного целого $n$;

$z_{(1, n)}=n !(1-i)$, если целое неотрицательное число $n$ имеет остаток 3 при делении на четыре, и $z_{(1, n)}=0$ в противном случае.

5.3. Случай нескольких неизвестных функций. Важно отметить, что обсуждаемая теорема сходимости формальных решений системы несложно обобщается на случай систем с несколькими неизвестными функциями $z_{1}, \ldots, z_{p}$.

Производные набора неизвестных функций $z_{1}, \ldots, z_{p}$ параметризуются точками произведения $Z=\mathbb{Z}_{\geqslant 0}^{n} \times\{1, \ldots, p\}$. Пусть $\prec_{\mathbb{Z}_{\geqslant 0}^{n}}-$ некоторый порядок на полугруппе $\mathbb{Z}_{\geqslant 0}^{n}$, удовлетворяющий условиям (a) и (b) из $\S 2$. Рассмотрим следующий полный порядок $\prec$ на множестве $Z$. Для любых элементов $(\alpha, i)$ и $(\beta, j)$ множества $Z$ элементы $\alpha$ и $\beta$ полугруппы $\mathbb{Z}_{\geqslant 0}^{n}$ сравниваются с помощью упорядочения $\prec_{\mathbb{Z}_{\geqslant 0}^{n}}$; если же они совпадают, сравниваются (как целые числа) индексы $i$ и $j$. В этом случае нетрудно доказать прямой аналог нашей теоремы, практически дословно повторяя рассуждения данной работы.

\section{ЛИТЕРАТУРА}

1. Riquier $C$. Les systèmes d'èquations aux derivées partielles. Gauthier-Villars, Paris, 1910.

2. Фиников C. П. Метод внешних форм Картана в дифференциальной геометрии. Теория совместности систем дифференциальных уравнений в полных дифференциалах и в частных производных. Гостехиздат, М.-Л., 1948.

3. Паламодов В. П. Дифференциальные операторы в классе сходящихся степенных рядов. Функц. анализ и его прил., 2, вып. 3, 58-69 (1968).

4. Хованский A. Г., Чулков C. П. Полином Гильберта для систем линейных дифференциальных уравнений в частных производных с аналитическими коэффициентами (работа представлена в журнал Изв. РАН).

5. Хованский A. Г., Чулков C. П. Полиномы Гильберта и Гильберта-Самюэля и уравнения в частных производных. Матем. заметки, 77, №1, 141-151 (2005).

6. Овсянников Л. В. Абстрактная форма теоремы Коши-Ковалевской и ее приложения. В сб.: Уравнения с частными производными (Труды конференции, Новосибирск, 1978). Наука, Сиб. отд., Новосибирск, 1980, с. 88-94.

7. Pate T. H. A direct iterative method for an abstract Cauchy-Kowalewsky theorem. Indiana Univ. Math J., 30, No. 3, 415-425 (1981).

8. Treves F. An abstract nonlinear Cauchy-Kovalevska theorem. Trans. Amer. Math. Soc., 150, 77-92 (1970).

9. Nirenberg L. An abstract form of the nonlinear Cauchy-Kowalewski theorem. In: Collection of articles dedicated to S. S. Chern and D. C. Spencer of their sixtieth birthdays. J. Differential Geom., 6, 561-576 (1972).

10. Nishida T. A note on a theorem of Nirenberg. J. Differential Geom., 12, 629-633 (1977).

11. Зайцева М. И. О совокупности упорядочений абелевой группы. УМН, 8, вып. 1, 135-137 (1953).

12. Trevisan $G$. Classificazione dei semplici ordinamenti di un gruppo libero commutativo con $N$ generatori. Rend. Sem. Mat. Univ. Padova, 22, 143-156 (1953).

Московский государственный университет

им. М. В. Ломоносова,

Поступило в редакцию

Независимый московский университет

1 сентября 2003 г.

e-mail: chulkov@mccme.ru 